\title{
Cáncer de mama en Jalisco. Análisis espacial de la mortalidad en 2010-2017
}

\author{
Igor M. Ramos-Herrera, ${ }^{1}$ Antonio Reyna-Sevilla, ${ }^{1 *}$ Miguel E. González Castañeda, ${ }^{2}$
} Juan D. Robles-Pastrana, ${ }^{2}$ Dulce D. Herrera-Echauri y César A. González-Rivera ${ }^{2}$

${ }^{1}$ Centro Universitario de Ciencias de la Salud; ${ }^{2}$ Centro Universitario de Ciencias Sociales y Humanidades, Universidad de Guadalajara, Jalisco, México

\section{Resumen}

Introducción: En 2017, el INEGI reportó 84142 defunciones por tumores malignos en México y la Organización Mundial de la Salud indicó que la tasa de mortalidad por cáncer de mama en 2018 fue de 11.2 por 100 mil mujeres. Objetivo: Mostrar la tendencia de la mortalidad por cáncer de mama en mujeres según municipio y región sanitaria de Jalisco en el periodo 2010-2017. Método: Estudio analítico en el que se estimaron tasas estandarizadas de mortalidad y riesgos relativos por municipio de residencia de 3873 mujeres. Se utilizó estadística espacial de dispersión y tendencia central. Resultados: La tasa de mortalidad aumentó de 10.7 a 13.0 por 100 mil mujeres en el periodo 2010-2017. Los valores más altos se encontraron en los municipios de Chapala (21.2) y Guadalajara (19.5), la tasa de mortalidad aumentó en cuatro de cada 10 municipios y el riesgo relativo fue hasta 50 veces mayor en algunos del occidente y centro de Jalisco. Conclusiones: Se observó un incremento de $1.0 \%$ anual, aunque territorialmente diferenciado. Los resultados representan una oportunidad para mejorar los procesos de detección y diagnóstico oportunos, así como para garantizar la cobertura de los servicios.

PALABRAS CLAVE: Neoplasias de mama. Riesgo relativo. Registros de mortalidad. Localización geográfica de riesgo.

\section{Breast cancer in Jalisco: a spatial analysis of mortality in the 2010-2017 period}

\begin{abstract}
Introduction: In 2017, INEGI reported 84,142 deaths from malignant tumors in Mexico, while the World Health Organization indicated that the breast cancer mortality rate in 2018 was 11.2 per 100,000 women. Objective: To show the trend of breast cancer mortality in women by municipality and health region of Jalisco in the 2010-2017 period. Method: Analytical study in which standardized mortality rates and relative risks of 3873 women were estimated by municipality of residence. Dispersion and central tendency spatial statistics were used. Results: The mortality rate increased from 10.7 to 13.0 per 100,000 women in the 2010-2017 period. The highest values were found in the municipalities of Chapala (21.2) and Guadalajara (19.5); the mortality rate increased in four out of every 10 municipalities, and relative risk was up to 50-fold higher in some of the western and central Jalisco municipalities. Conclusions: An annual increase of $1.0 \%$ was observed, although it was territorially differentiated. The results represent an opportunity to improve timely detection and diagnostic processes, as well as to guarantee the coverage of services.
\end{abstract}

KEY WORDS: Breast neoplasms. Relative risk. Mortality records. Geographical risk location.

Correspondencia:

*Antonio Reyna-Sevilla

E-mail: gs.antonioreyna@gmail.com
Fecha de recepción: 19-09-2019

Fecha de aceptación: 23-01-2020

DOI:10.24875/GMM.20005546
Gac Med Mex. 2020;156:542-548

Disponible en PubMed

www.gacetamedicademexico.com

0016-3813/@ 2020 Academia Nacional de Medicina de México, A.C. Publicado por Permanyer. Este es un artículo open access bajo la licencia CC BY-NC-ND (http://creativecommons.org/licenses/by-nc-nd/4.0/). 


\section{Introducción}

Uno de los mayores desafíos en términos de salud pública en México son las enfermedades cronicodegenerativas, como las neoplasias., ${ }^{1,2}$ En 2017, según el Instituto Nacional de Estadística y Geografía (INEGI), se atribuyeron 84142 defunciones a tumores malignos, es decir, $12 \%$ de mortalidad en la población general. ${ }^{3}$ Entre ellos, el cáncer de mama (CaMa) en mujeres mexicanas representa la causa de muerte más frecuente desde 2006., En parte, lo anterior podría ser explicado por el número de diagnósticos que se realizan cada año; hasta cuatro de cada 10 casos en mujeres entre 30 y 50 años resultan ser CaMa. ${ }^{5}$ Además, la Organización Panamericana de la Salud indica que en 2018 la tasa de mortalidad por CaMa en México fue de 11.2 por 100 mil mujeres, ${ }^{6}$ lo que ciertamente representa una disminución importante a la reportada en 2012: 17.1 defunciones por 100 mil mujeres. ${ }^{7}$ Sin embargo, aun con los esfuerzos para reducir tal magnitud, que algunos autores atribuyen a las mejoras en los procesos de diagnóstico o acceso a tratamiento oncológico, ${ }^{2}$ la mayoría de los casos en México se diagnostica en fases avanzadas (estadios clínicos II-b a IV), ,-10 con diferencias entre los estados o municipios, lo cual incrementa el riesgo de mortalidad por CaMa en las mujeres. ${ }^{8,11}$

Este panorama epidemiológico ha impulsado, entre otras cosas, el desarrollo de investigaciones enfocadas en determinar la magnitud y tendencias del CaMa en México, pero en menor proporción las de variaciones geográficas y focalización de la mortalidad. Se conocen estudios epidemiológicos que incluyen el análisis de datos correspondientes a largos periodos, por ejemplo, entre siete y 31 años, ${ }^{2,5,12-17}$ desarrollados en los estados ${ }^{5,13-15,18}$ o municipios; ${ }^{; 3}$ aunque no todos referentes a CaMa. Entre las principales contribuciones de esas investigaciones destacan estimaciones actualizadas sobre la mortalidad, evaluación del progreso de los servicios de prevención, detección y tratamiento, establecidos en la NOM-041-SSA2-2011; ${ }^{19}$ así como la determinación de necesidades de intervención y mejora de dichos aspectos. ${ }^{2,5}$

Sin embargo, significativamente existen menos análisis que incorporen una perspectiva territorial, por ejemplo, para reconocer la distribución y variación de patrones espaciales asociados con el CaMa, identificar lugares donde se requiere anticipar y garantizar la cobertura de servicios de salud de mujeres en riesgo de padecerlo, o bien, focalizar acciones de prevención secundaria, ${ }^{20}$ como lo establece la NOM041-SSA2-2011, ${ }^{19}$ y con ello mejorar los procesos involucrados en la detección temprana, diagnóstico oportuno y tratamientos especializados.

En este sentido, la epidemiología espacial, que incluye una perspectiva territorial en sus herramientas de análisis, puede contribuir a generar evidencia complementaria a los estudios sobre tendencias de mortalidad por CaMa mediante el análisis de la distribución espacio-temporal de enfermedades o causas de muerte, mapeo de lugares de mayor riesgo o identificación de patrones en diferentes escalas territoriales. ${ }^{21,22}$ Este aspecto resulta de suma importancia para respaldar la toma de decisiones ${ }^{22}$ y la asignación de recursos (materiales y humanos) asociados con la prevención secundaria, ${ }^{14}$ con la finalidad de reducir, en última instancia, desigualdades en salud. ${ }^{22}$

\section{Objetivo}

Mostrar desde un enfoque de la epidemiología espacial y salud pública, la tendencia espacio-temporal en años recientes del riesgo que tienen las mujeres de Jalisco de morir por CaMa, así como analizar las diferencias geográficas de las tasas de mortalidad por municipio y región sanitaria en el estado durante el periodo 2010-2017.

\section{Método}

Se llevó a cabo un diseño analítico y transversal, dada la fuente secundaria consultada para la obtención de datos y la agrupación de estos en áreas geográficas para análisis y comparación. En la primera etapa se obtuvieron estadísticas de mortalidad de 2010 a 2017 relacionadas con CaMa en mujeres según municipio de residencia, obtenidas del Registro Estatal de Cáncer de la Secretaría de Salud de Jalisco. Dichos datos fueron georreferenciados considerando los 125 municipios en Jalisco, según el Marco Geoestadístico Nacional del INEGI, ${ }^{23}$ así como las 13 regiones sanitarias que integran dicha demarcación territorial, de acuerdo con datos oficiales de esa Secretaría.

Con base en los datos georreferenciados, una segunda etapa consistió en estimar tasas de mortalidad estandarizadas por municipio y año. ${ }^{24}$ Para ello se utilizó el método empírico de suavizado de Bayes, con la finalidad de evitar una representación espuria en la magnitud y distribución geográfica de las tasas, cuyo efecto se produce por las diferencias en el tamaño de la población según la unidad espacial de 
análisis utilizada (municipio).. ${ }^{24}$ En dicho cálculo se utilizaron proyecciones oficiales de población femenina mayor de 20 años por municipio a través de la herramienta Cubos Dinámicos de la Dirección General de Información en Salud. ${ }^{25}$ Adicionalmente, se estimaron otros indicadores para el análisis de la mortalidad por CaMa: riesgo relativo y razón de incremento (RI) entre 2010 y 2017; ambos fueron representados en mapas por municipio y región sanitaria.

En una tercera etapa se estimó la variabilidad y tendencia (aumento o disminución) de la tasa estandarizada de mortalidad mediante regresión lineal. Además, para identificar la magnitud y diferencias geográficas de las tasas calculadas se utilizó estadística espacial, esto es, se obtuvieron medidas de dispersión, tendencia central y direccional (elipse de desviación estándar) para determinar en qué municipios se concentraron los valores más altos, cuáles fueron los que registraron incremento o decremento de 2010 a 2017, y para definir áreas de mayor riesgo de mortalidad por CaMa.

\section{Resultados}

En Jalisco, según la fuente utilizada, se registraron 3873 defunciones por CaMa de 2010 a 2017, reportadas únicamente en 119 municipios (95.2\%), lo que se traduce en una tasa cruda de 12.2 por 100 mil mujeres mayores de 20 años. En la Figura 1 se muestra el análisis de regresión lineal, donde destaca una tendencia ascendente en los fallecimientos por CaMa $(p<0.05)$; además, se identificó un incremento total de $17 \%$ según la tasa de mortalidad correspondiente a 2010-2017.

Por otra parte, en la Figura 2 es posible observar la magnitud y distribución geográfica de la tasa de mortalidad según municipio de residencia al momento de la defunción (2010-2017), cuyos valores oscilaron entre 5.3 y 21.2 por 100 mil mujeres mayores de 20 años. Como resultado, los municipios de Chapala y Guadalajara presentaron una tasa estandarizada hasta dos veces mayor que el promedio municipal (valores superiores atípicos): 21.2 y 19.5 defunciones por 100 mil mujeres mayores de 20 años, respectivamente. Dichos municipios pertenecen a las regiones sanitarias La Barca y Guadalajara, localizadas en la zona central del estado.

En el mapa de la Figura 3 se presenta la variabilidad geográfica de la tasa de mortalidad entre 2010 y 2017 por municipio y región sanitaria (RI); como resultado, se puede apreciar que en 68 municipios (54.4\%) la mortalidad disminuyó, en otros siete municipios (5.6\%) no hubo cambio, mientras que la mortalidad aumentó hasta 90 veces en los 50 municipios

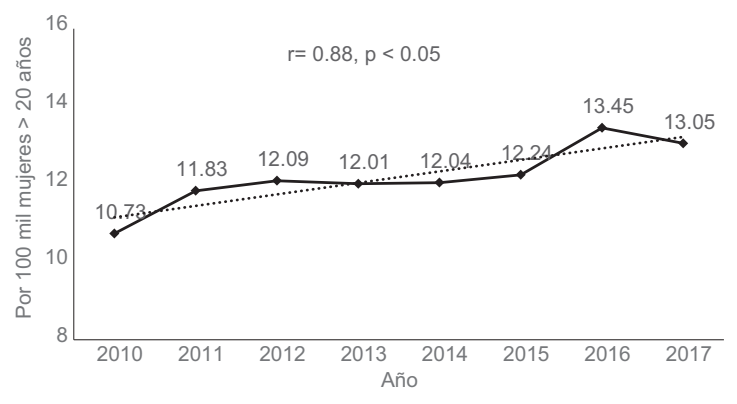

Figura 1. Tendencia en la tasa estandarizada de mortalidad por cáncer de mama en Jalisco, 2010-2017. Fuente: elaboración propia con base en estadísticas de mortalidad por cáncer de mama en mujeres atendidas en la Secretaría de Salud de Jalisco.

restantes (40\%); esto último se registró principalmente en las regiones sanitarias del centro (Zapopan, Guadalajara, Tonalá) y en dirección al noreste de Jalisco (Tepatitlán, Lagos de Moreno).

Mediante la estimación del RR de mortalidad por CaMa según área de residencia (Figura 4) se identificaron 50 municipios (40\%) en los cuales dicho indicador fue $>1$, pero el RR aumentó más de 50 veces particularmente en 12 municipios (9.6\%). Dichos resultados se pueden apreciar en el mapa de la Figura 4, desde la zona occidente (Región Sanitaria VIII-Puerto Vallarta Costa Norte) hacia el noreste de Jalisco (Región Sanitaria III-Tepatitlán), lo cual forma una franja que atraviesa transversalmente todo el estado. Más aún, el análisis mediante la elipse de desviación estándar corrobora esta tendencia espacial (Figura 5), lo cual indica la zona donde la tasa de mortalidad por CaMa fue más alta en comparación con otros municipios; de hecho, dicha delimitación explica $83.6 \%$ $(n=3240)$ de las defunciones registradas en el periodo de estudio (isoconcentración). Adicionalmente, en el mapa de la Figura 5 se pueden observar los resultados de la tendencia espacial, lo que implica una delimitación asociada con baja y alta mortalidad por CaMa, independientemente de los límites político-administrativos (municipios y regiones sanitarias) en Jalisco, con orientación suroeste-noreste.

\section{Discusión}

Entre 2010 y 2017, la mortalidad por CaMa en Jalisco aumentó $17 \%$. Sin embargo, únicamente en cuatro de cada 10 municipios se observó esta tendencia, cuya ubicación se identificó desde el occidente hacia el centro del estado, donde el RR aumentó hasta $50 \%$. Por ello, consideramos que la concentración y 


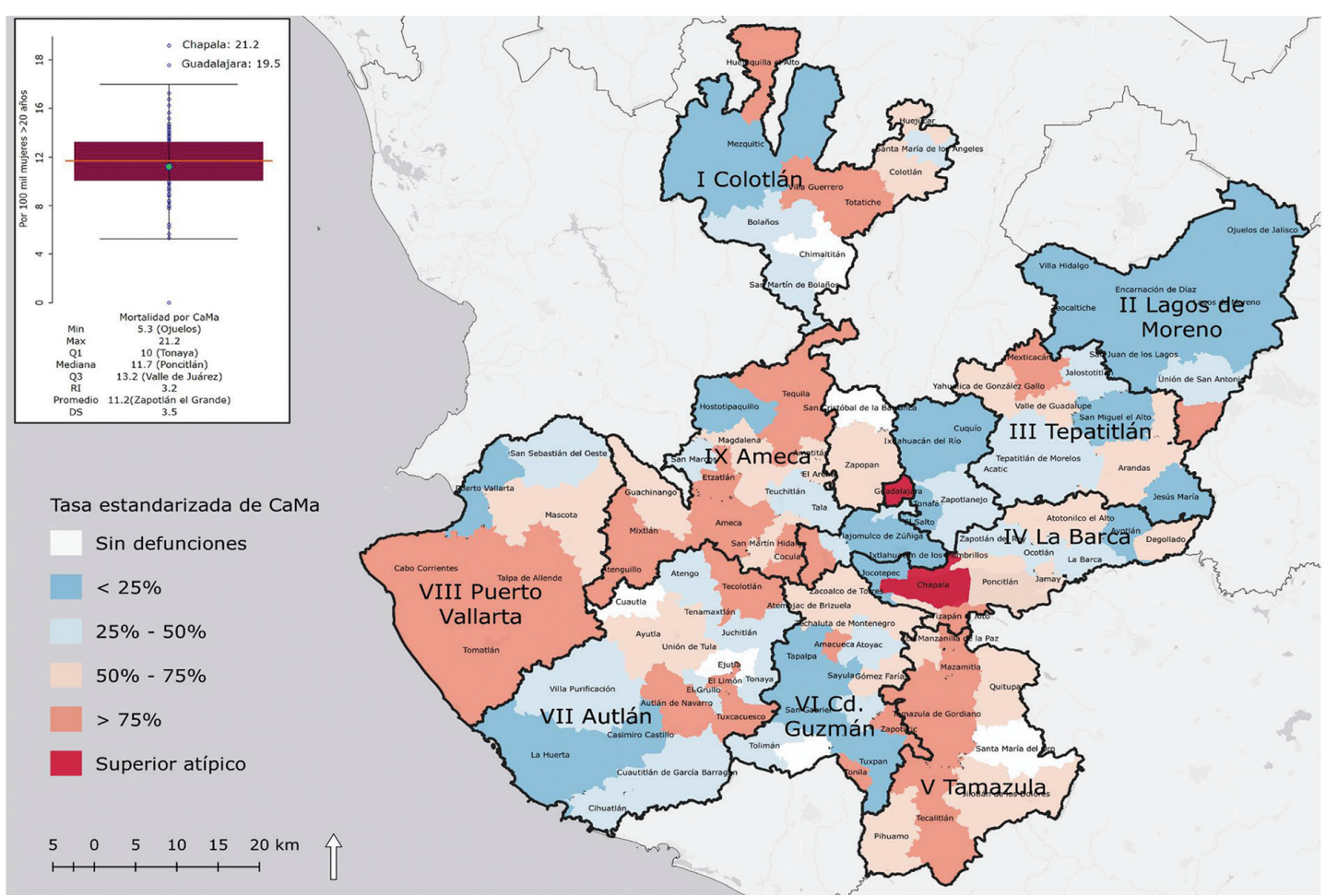

Figura 2. Magnitud y distribución geográfica de la tasa estandarizada de mortalidad por cáncer de mama según municipio de residencia, Jalisco 2010-2017. Fuente: elaboración propia con base en estadísticas vitales de mortalidad por cáncer de mama en mujeres atendidas en la Secretaría de Salud de Jalisco, así como con base en el Marco Geoestadístico Estatal y Municipal de Jalisco. ${ }^{23}$

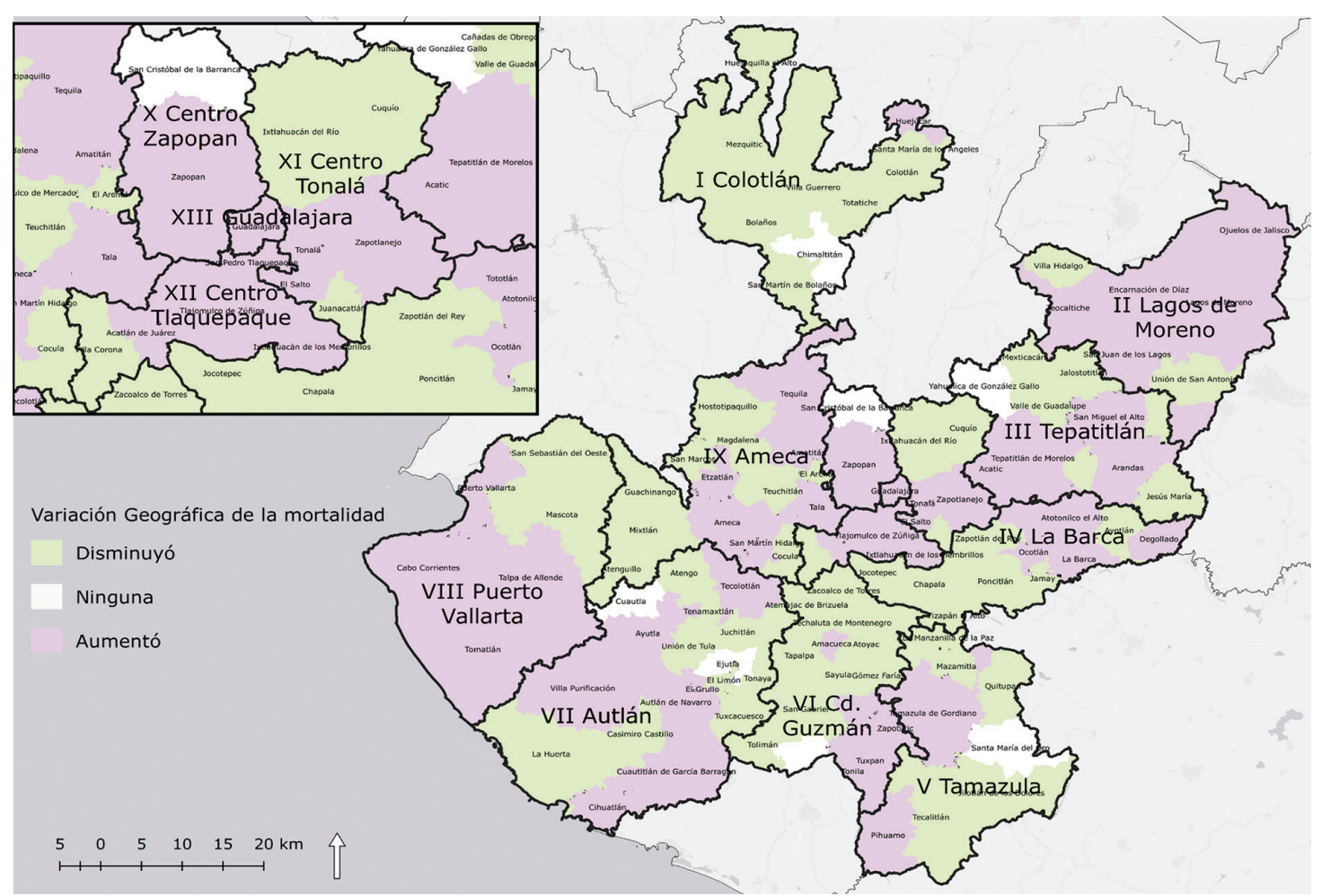

Figura 3. Variación geográfica del incremento de la mortalidad por cáncer de mama entre 2010 y 2017 según municipio de residencia, Jalisco. Fuente: elaboración propia con base en estadísticas vitales de mortalidad por cáncer de mama en mujeres atendidas en la Secretaría de Salud de Jalisco, así como con base en el Marco Geoestadístico Estatal y Municipal de Jalisco. ${ }^{23}$ 


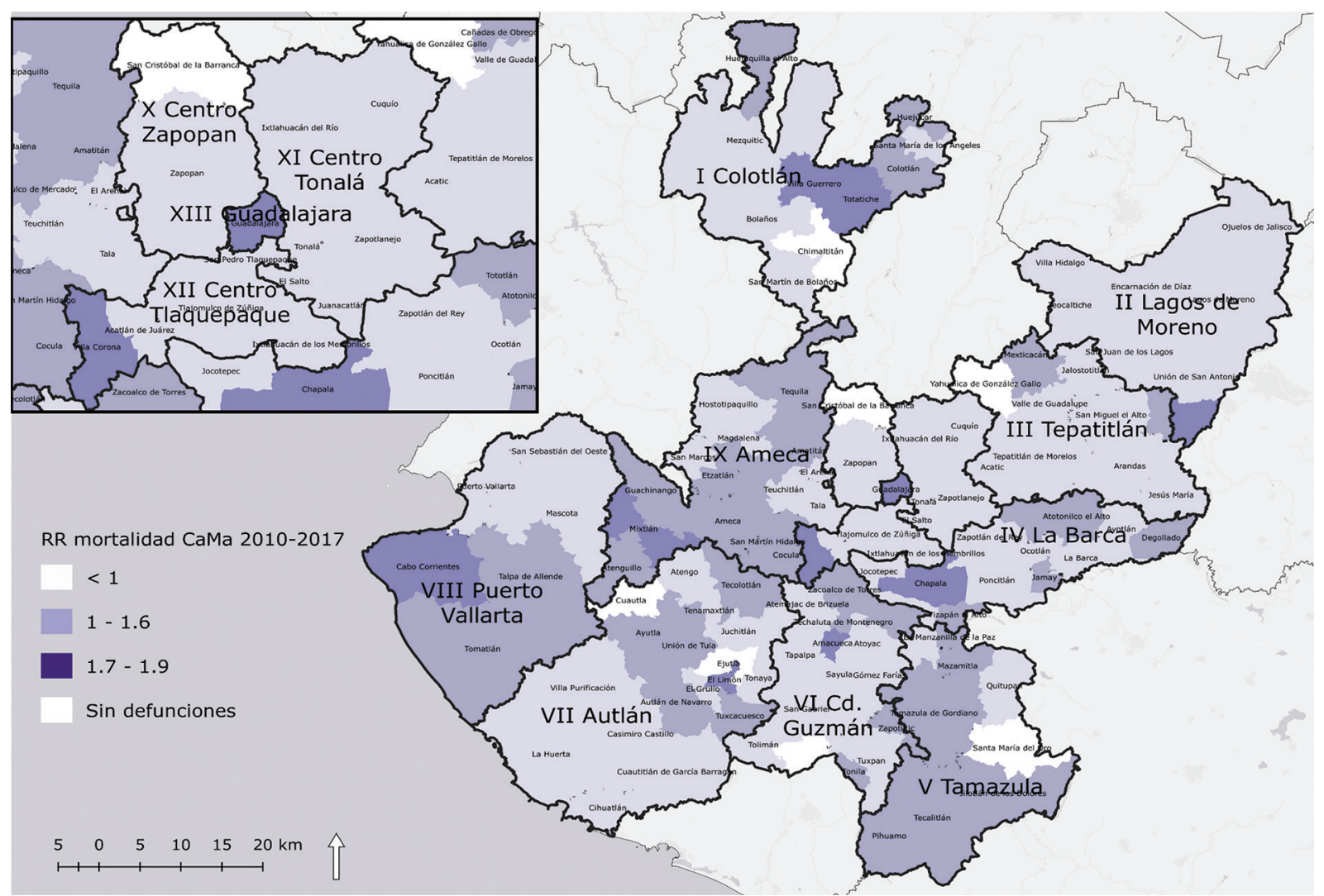

Figura 4. Distribución geográfica del riesgo relativo de mortalidad por cáncer de mama según municipio de residencia, Jalisco $2010-2017$. Fuente: elaboración propia con base en estadísticas vitales de mortalidad por cáncer de mama en mujeres de mama en mujeres atendidas en Salud de Jalisco, así como con base en el Marco Geoestadístico Estatal y Municipal de Jalisco. ${ }^{23}$

dispersión de la mortalidad por CaMa representa una oportunidad para reconocer los municipios donde se requiere mejorar los procesos de detección y diagnóstico clínico oportunos, como lo establece la NOM-041SSA2-2011, ${ }^{19}$ dado que constituyen focos que explican la mortalidad en determinados contextos ${ }^{8,10}$ en los cuales puede existir mayor cercanía a los servicios de diagnóstico y tratamiento del CaMa.

Aun cuando la Organización Mundial de la Salud reportó una disminución de la mortalidad por $\mathrm{CaMa}$ en mujeres mexicanas, recientemente se ha evidenciado un incremento real. 2,10,12,13,15-17 Como ejemplo, de 1990 a 2013, la mortalidad aumentó 15 \% en Jalisco, ${ }^{5}$ el cual, incluso, ha sido uno de los estados con tasas más elevadas en comparación con otros de la República Mexicana. ${ }^{15}$ De tal forma, dicho panorama es consistente con los resultados aquí reportados, aun cuando desde 2007 se ofrece atención gratuita y tratamientos óptimos en todos los estadios de $\mathrm{CaMa}$ como parte del programa Gastos Catastróficos del Seguro Popular. ${ }^{8}$ Por esa razón, consideramos que el incremento de la mortalidad en Jalisco podría estar relacionado con deficiencias en los procesos de detección y diagnóstico clínico de CaMa, aunado a la baja respuesta de las mujeres a los programas preventivos del $\mathrm{CaMa}^{12}$ o a mayor disponibilidad $\mathrm{y}$ acceso a servicios de salud;:,13 más allá de los factores de riesgo conocidos. ${ }^{5,8}$

La Encuesta Nacional de Salud y Nutrición ${ }^{26}$ reportó en Jalisco una de las coberturas de mamografía como método de detección precoz- más bajas del país, $29.3 \%$ menos en comparación con estados del norte, donde la proporción de mujeres que se realizan el estudio de tamizaje fue cuatro de cada $10 .{ }^{26}$ Por ello, es de suponer que el estadio clínico al momento del diagnóstico generalmente se realice en etapas avanzadas de la enfermedad (estadios IIb a IV), como sucede en otros estados de México, ${ }^{8-10}$ lo que limita el pronóstico y las posibilidades de supervivencia., ${ }^{428}$ Así, es posible suponer que el estadio clínico y el proceso de detección podrían explicar la magnitud de la mortalidad por CaMa reportada en Jalisco en años recientes. ${ }^{12}$ Según Roche, Skinner \& Weinstein,$^{11}$ los diagnósticos en fases avanzadas son consecuencia 


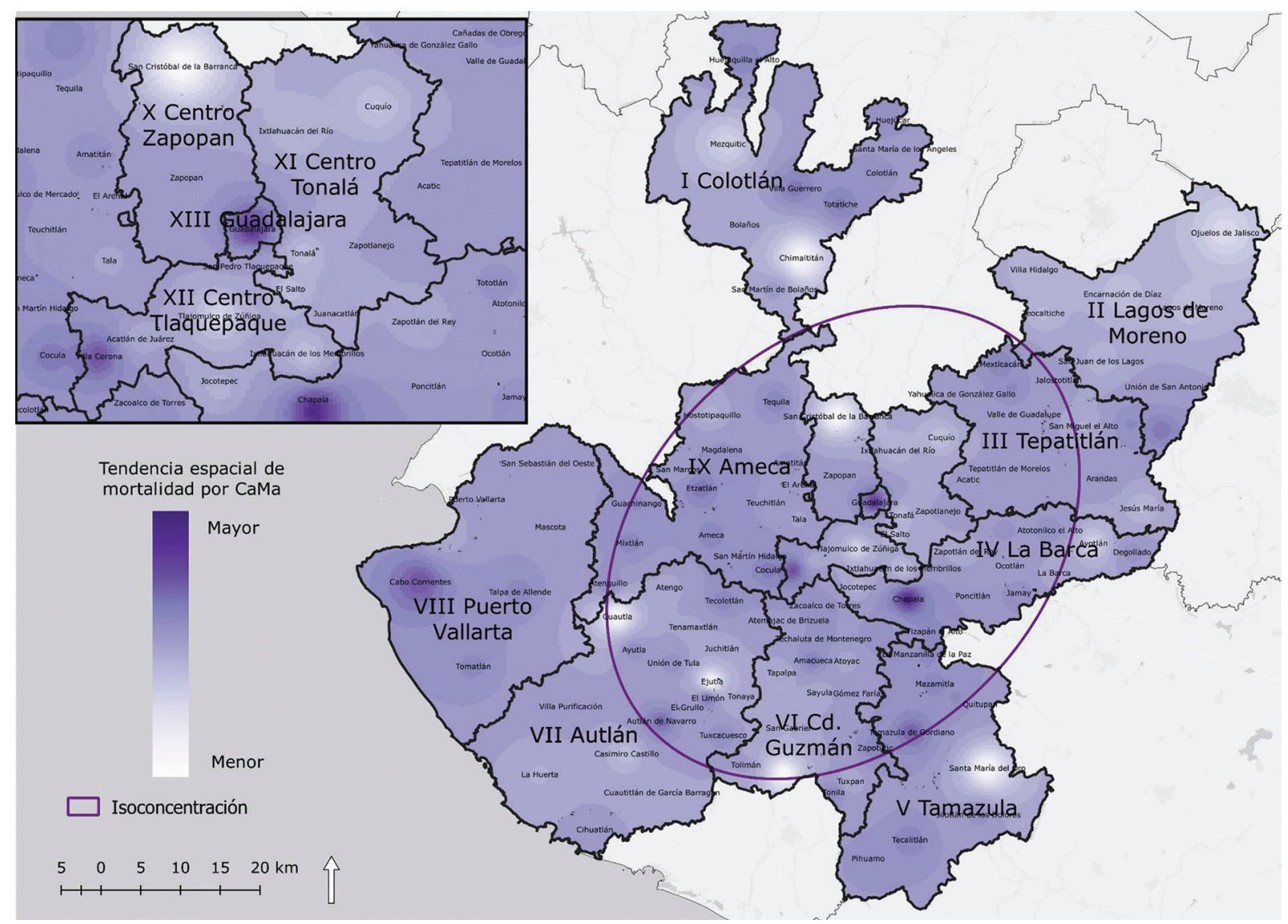

Figura 5. Tendencia espacial de la mortalidad por cáncer de mama en el contexto de los municipios y regiones sanitarias de Jalisco. Fuente: elaboración propia con base en estadísticas vitales de mortalidad por cáncer de mama en mujeres atendidas en la Secretaría de Salud de Jalisco, así como con base en el Marco Geoestadístico Estatal y Municipal de Jalisco. ${ }^{23}$

de la poca efectividad de las acciones dirigidas a detectar tempranamente la enfermedad. Por lo tanto, nuestros resultados definen, en principio, a Chapala y Guadalajara como los municipios donde se requiere evaluar las acciones destinadas a prevenir, detectar y ofrecer tratamiento especializado para reducir la carga de mortalidad por CaMa.

Una de las estrategias más efectivas para el control y vigilancia epidemiológica del CaMa, según el reporte mundial sobre cáncer, es la prevención secundaria, la cual se refiere a detectar la enfermedad en etapas premalignas. ${ }^{20}$ En este sentido, nuestros resultados podrían servir de base para organizar programas de detección, mejorar procesos de diagnóstico y garantizar un tratamiento oportuno, como lo establece la NOM-041-SSA2-2011, ${ }^{19}$ en municipios de mayor riesgo desde el occidente hasta la zona centro de Jalisco. Ello con la finalidad de minimizar los problemas relacionados con la mortalidad por CaMa, como el retraso en el diagnóstico, el inicio del tratamiento oncológico, o bien, las desigualdades geográficas.
Por otra parte, es necesario considerar algunas limitaciones relacionadas con el uso de datos de una fuente secundaria, en los cuales podría existir subregistro de las defunciones analizadas, si bien la calidad en el registro de defunciones ha mejorado significativamente en México en los últimos años. ${ }^{2}$ De hecho, la información presentada puede servir para dilucidar el desafío que enfrenta la Secretaría de Salud de Jalisco en torno a la magnitud y variabilidad de la mortalidad por CaMa en los municipios, aunado a la carga financiera que implica el diagnóstico y tratamiento. Otra limitante estriba en el análisis de datos retrospectivos de varios años, por lo que su interpretación debe realizarse con cautela.

En conclusión, destacamos tres aspectos importantes:

- Primero, se identificaron municipios donde la mortalidad se incrementó en el periodo 20102017 (40\%), desde el centro hacia el noreste de Jalisco, en los cuales se sugiere evaluar los procesos involucrados en la prevención, detección, 
diagnóstico clínico y disponibilidad de tratamientos especializados.

- Segundo, se identificaron municipios (9.6\%), desde el occidente hacia el centro, en los cuales es necesario promover con mayor énfasis servicios de mastografía para detectar precozmente la enfermedad, dado que, según el área de residencia, el RR se incrementó hasta 50 veces.

- Finalmente, mediante el uso de datos georreferenciados y herramientas geográficas, se identificaron áreas de riesgo o prioritarias que requieren atención para acometer desigualdades en la atención de las mujeres.

\section{Agradecimientos}

Los autores agradecen a los funcionarios de la Secretaría de Salud de Jalisco por proporcionar los datos necesarios para la investigación.

\section{Conflicto de intereses}

Los autores declaran no tener conflictos de intereses.

\section{Financiamiento}

Los autores no recibieron patrocinio para elaborar este artículo.

\section{Responsabilidades éticas}

Protección de personas y animales. Los autores declaran que para esta investigación no se realizaron experimentos en seres humanos ni en animales.

Confidencialidad de los datos. Los autores declaran que en este artículo no aparecen datos de pacientes.

Derecho a la privacidad y consentimiento informado. Los autores declaran que en este artículo no aparecen datos de pacientes.

\section{Bibliografía}

1. Lozano R, Gómez H, Pelcastre B, Ruelas M, Montañez J, Campuzano J, et al. Carga de la enfermedad en México, 1990-2010. Nuevos resultados y desafíos. México: Centro de Investigación en Sistemas de Salud; 2013.
2. Torres-Sánchez LE, Rojas-Martínez R, Escamilla-Núñez C, de la Vara-Salazar E, Lazcano-Ponce E. Tendencias en la mortalidad por cáncer en México de 1980 a 2011. Salud Publica Mex. 2014;56:473-491.

3. Instituto Nacional de Estadística y Geografía [sitio web]. México: Estadísticas a propósito del Día Mundial contra el Cáncer (4 de febrero). Datos nacionales. [Comunicado de prensa]; 2018.

4. De la Vara-Salazar E, Suárez-López L, Ángeles-Llerenas A, Torres-Mejía G, Lazcano-Ponce E. Mortalidad por cáncer de mama en México: niveles, evolución y tendencias 1980-2009. En Lazcano-Ponce E, Escudero-de los Ríos $\mathrm{P}$, Uscanga-Sánchez $\mathrm{S}$, editores. Cáncer de mama. Diagnóstico, tratamiento, prevención y control. México: Instituto Nacional de Salud Pública; 2014.

5. Gómez-Dantés H, Lamadrid-Figueroa H, Cahuana-Héctor L, Silverman-Retana O, Montero P, González-Robledo MC, et al. The burden of cancer in México, 1990-2013. Salud Publica Mex. 2016:58(2):118-131.

6. Organización Panamericana de la Salud [sitio web]. EE. UU.: Situación de salud en las Américas: indicadores básicos; 2018.

7. Centro Nacional de Equidad de Género y Salud Reproductiva. Programa de acción específico. Prevención y control del cáncer de la mujer 20132018. México: Secretaría de Salud; 2014.

8. Cárdenas-Sánchez J, Erazo-Valle Solís AA, Arce-Salinas C, Bargalló-Rocha JE, Bautista-Piña V, Cervantes-Sánchez G, et al., editores. Consenso mexicano sobre diagnóstico y tratamiento del cáncer mamario. México: Elsevier. 2017.

9. Maffuz-Aziz A, Labastida-Almendaro S, Espejo-Fonseca A, Rodríguez-Cuevas S. Características clinicopatológicas del cáncer de mama en una población de mujeres en México. Cir Ciruj. 2017;85:201-207.

10. Chávarri-Guerra Y, Villarreal-Garza C, Liedke PER, Knaul F, Mohar A Finkelstein DM, et al. Breast cancer in Mexico: a growing challenge to health and the health system. Lancet Oncol. 2012;13:e335-e343.

11. Roche LM, Skinner R, Weinstein RB. Use of a geographic information system to identify and characterize areas with high proportions of distant stage breast cancer. J Public Heal Manag Pract. 2002;8:26-32.

12. Ramos-Herrera I, González-Castañeda E, Reyna-Sevilla A, Robles-Pastrana JD, García-Sandoval C. Mortalidad por cáncer de mama en Jalisco: un análisis espacio temporal a nivel municipal. UniverSalud. 2016;12:37-54.

13. Aguayo AG, Jiménez PL, Celis RA, Canales MJ. Mortalidad por cáncer de mama. Tendencia y distribución geográfica, 1981-2001. Rev Med Inst Mex Seguro Soc. 2008;46:367-374.

14. Palacio ML, Lazcano PE, Allen LB, Hernández AM. Diferencias regionales en la mortalidad por cáncer de mama y cérvix en México entre 1979 y 2006. Salud Publica Mex. 2009;51:208-219.

15. De la Vara-Salazar E, Suárez-López L, Ángeles-Llerenas A, Torres-Mejía G, Lazcano-Pone E. Tendencias de la mortalidad por cáncer de mama en México, 1980-2009. Salud Publica Mex. 2011;53:385-393.

16. Anaya RM, Vallejo RV, Flores ML, Pérez SM. Female breast cancer incidence and mortality in Mexico, 2000-2010. Asian Pac J Cancer Prev. 2014;15:1477-1479.

17. Bosetti C, Rodríguez T, Chatenoud L, Bertuccio P, Levi F, Negri E, et al. Trends in cancer mortality in Mexico, 1981-2007. Eur $J$ Cancer Prev. 2011;20:355-363.

18. Navarrete-Valero C, Navarrete-Vázquez C. Mortalidad por cáncer mamario, prostático y cervicouterino, años perdidos y costos de los programas. México, 2013 a 2016. Gac Med Mex. 2018;154:665-670.

19. Norma Oficial Mexicana NOM-041-SSA2-2011 para la prevención, diagnóstico, tratamiento, control y vigilancia epidemiológica del cáncer de mama. México: Diario Oficial de la Federación; 2011.

20. Stewart BW, Wild CW. World Cancer Report 2014. EE. UU.: World Health Organization; 2014

21. Roquette R, Painho M, Nunes B. Spatial epidemiology of cancer: a review of data sources, methods and risk factors. Geospat Health. 2017;12:504.

22. Kirby RS, Delmelle E, Eberth JM. Advances in spatial epidemiology and geographic information systems. Ann Epidemiol. 2017;27:1-9

23. Instituto Nacional de Estadística y Geografía [sitio web]. México: Marco geoestadístico, junio; 2018.

24. Center for Spatial Data Science [sitio web]. Illinois, Chicago: GeoDa: an introduction to Spatial Data Analysis; 2018.

25. Secretaría de Salud [sitio web]. México: Dirección General de Información en Salud. Población. Cubos Dinámicos; 2019.

26. Instituto Nacional de Salud Pública. Encuesta Nacional de Salud y Nutrición 2012. Resultados Nacionales. Síntesis ejecutiva. México: Instituto Nacional de Salud Pública; 2012. 